\title{
Numerical Study for the Fractional Differential Equations Generated by Optimization Problem Using Chebyshev Collocation Method and FDM
}

\author{
M. M. Khader ${ }^{1,2, *}$, N. H. Sweilam ${ }^{3}$ and A. M. S. Mahdy ${ }^{4}$ \\ ${ }^{1}$ Department of Mathematics and Statistics, College of Science, Al-Imam Mohammed Ibn Saud Islamic University, Riyadh, Saudi \\ Arabia \\ ${ }^{2}$ Department of Mathematics, Faculty of Science, Benha University, Benha, Egypt \\ ${ }^{3}$ Department of Mathematics, Faculty of Science, Cairo University, Giza, Egypt \\ ${ }^{4}$ Department of Mathematics, Faculty of Science, Zagazig University, Zagazig, Egypt
}

Received: 3 Feb. 2013, Revised: 4 Jun. 2013, Accepted: 5 Jun. 2013

Published online: 1 Sep. 2013

\begin{abstract}
This paper is devoted with numerical solution of the system fractional differential equations (FDEs) which are generated by optimization problem using the Chebyshev collocation method. The fractional derivatives are presented in terms of Caputo sense. The application of the proposed method to the generated system of FDEs leads to algebraic system which can be solved by the Newton iteration method. The method introduces a promising tool for solving many systems of non-linear FDEs. Two numerical examples are provided to confirm the accuracy and the effectiveness of the proposed methods. Comparisons with the fractional finite difference method (FDM) and the fourth order Runge-Kutta (RK4) are given.
\end{abstract}

Keywords: Non-linear programming, penalty function, dynamic system, Caputo fractional derivatives, Chebyshev approximations, finite difference method, Runge-Kutta method.

\section{Nomenclature}

$D^{\alpha}$ : The Caputo fractional derivative of order $\alpha$;

$\mathbb{N}$ : $\quad$ The set of all nature numbers;

$\lceil\alpha\rceil$ : The ceiling function to denote the smallest integer greater than or equal to $\alpha$;

$\Re$ : $\quad$ The set of all real numbers;

$\nabla h(x)$ : The gradient of the function $h(x)$;

$\mu$ : $\quad$ An auxiliary penalty variable;

$\theta: \quad$ A constant;

$T_{n}(x)$ : The Chebyshev polynomial of degree $n$;

\section{Introduction}

In last decades, fractional calculus has drawn a wide attention from many physicists and mathematicians, because of its interdisciplinary application and physical meaning $[1,2]$. Fractional calculus deals with the generalization of differentiation and integration of non-integer order. Fractional differential equations have been the focus of many studies due to their frequent appearance in various applications in fluid mechanics, biology, physics and engineering [3]. Consequently, considerable attention has been given to the solutions of FDEs and integral equations of physical interest. Most FDEs do not have exact analytical solutions, so approximate and numerical techniques $[4,5]$ must be used. Several numerical methods to solve FDEs have been given such as, homotopy perturbation method [5], homotopy analysis method [6], collocation method [7, 14] and others [12].

Representation of a function in terms of a series expansion using orthogonal polynomials is a fundamental concept in approximation theory and form the basis of the solution of differential equations $[15,16]$. Chebyshev polynomials are widely used in numerical computation. One of the advantages of using Chebyshev polynomials as a tool for expansion functions is the good representation of smooth functions by finite Chebyshev expansion provided that the function $y(x)$ is infinitely differentiable.

\footnotetext{
*Corresponding author e-mail: mohamedmbd@yahoo.com
} 
The coefficients in Chebyshev expansion approach zero faster than any inverse power in $n$ as $n$ goes to infinity.

Optimization theory is aimed to find out the optimal solution of problems which are defined mathematically from a model arise in wide range of scientific and engineering disciplines. Many methods and algorithms have been developed for this purpose since 1940. The penalty function methods are classical methods for solving non-linear programming (NLP) problem [17, 18]. Also, differential equation methods are alternative approaches to find solutions to these problems. In this type of methods the optimization problem is formulated as a system of ordinary differential equations so that the equilibrium point of this system converges to the local minimum of the optimization problem [19,21].

In this article, we will compare our approximate solution with those numerical obtained using the implicit finite difference method. It has been shown that FDM is a powerful tool for solving various kinds of problems [22, 23]. Also, this technique reduces the problem to a system of algebraic equations. Many authors have pointed out that the FDM can overcome the difficulties arising in the calculation of some numerical methods, such as, finite element method.

The main aim of the presented paper is concerned with the application of the Chebyshev collocation method and fractional finite difference method to obtain the numerical solution of the system of FDEs which is generated from the non-linear programming problems and study the convergence analysis of the proposed method.

The structure of this paper is arranged in the following way: In section 2, we introduce some basic definitions about Caputo fractional derivatives, the definition of the optimization problem and its generated system of FDEs. In section 3, we derive an approximate formula for fractional derivatives using Chebyshev series expansion and estimate an upper bound of the resulting error of the proposed formula. In section 4, numerical examples are given to solve the system of FDEs which obtained from the non-linear programming problem and show the accuracy of the presented methods. Finally, in section 5, the paper ends with a brief conclusion and some remarks.

\section{Preliminaries and notations}

In this section, the formulation of the optimization problem and its corresponding system of FDEs are given and we present some necessary definitions and mathematical preliminaries of the fractional calculus theory required for our subsequent development.

\subsection{The fractional derivative in the Caputo sense}

The Caputo fractional derivative operator $D^{\alpha}$ of order $\alpha$ is defined in the following form

$$
D^{\alpha} f(x)=\frac{1}{\Gamma(m-\alpha)} \int_{0}^{x} \frac{f^{(m)}(\xi)}{(x-\xi)^{\alpha-m+1}} d \xi, \quad \alpha>0,
$$

where $m-1<\alpha \leq m, m \in \mathbb{N}, x>0$.

Similar to integer-order differentiation, Caputo fractional derivative operator is linear

$$
D^{\alpha}\left(c_{1} p(x)+c_{2} q(x)\right)=c_{1} D^{\alpha} p(x)+c_{2} D^{\alpha} q(x),
$$

where $c_{1}$ and $c_{2}$ are constants. For the Caputo's derivative we have

$$
D^{\alpha} C=0, \quad \mathrm{C} \text { is a constant, }
$$

$$
D^{\alpha} x^{n}= \begin{cases}0, & \text { for } n \in \mathbb{N}_{0} \text { and } n<\lceil\alpha\rceil \\ \frac{\Gamma(n+1)}{\Gamma(n+1-\alpha)} x^{n-\alpha}, & \text { for } n \in \mathbb{N}_{0} \text { and } n \geq\lceil\alpha\rceil\end{cases}
$$

We use the ceiling function $\lceil\alpha\rceil$ to denote the smallest integer greater than or equal to $\alpha$ and $\mathbb{N}_{0}=\{0,1,2, \ldots\}$. Recall that for $\alpha \in \mathbb{N}$, the Caputo differential operator coincides with the usual differential operator of integer order.

For more details on fractional derivatives definitions and their properties see ( [15], [24]).

\subsection{Optimization problem and its corresponding system of FDEs}

Consider the non-linear programming problem with equality constraints defined by

$$
\text { minimize } f(x), \quad \text { subject to } x \in M,
$$

with $M=\left\{x \in \mathfrak{R}^{n}: h(x)=0\right\}$, where $f: \mathfrak{R}^{n} \rightarrow \mathfrak{R}$ and $h=\left(h_{1}, h_{2}, \ldots, h_{p}\right)^{T}: \mathfrak{R}^{n} \rightarrow \mathfrak{R}^{p}(p \leq n)$. It is assumed that the functions in the problem are at least twice continuously differentiable, that a solution exists, and that $\nabla h(x)$ has full rank. To obtain a solution of (3), the penalty function method solves a sequence of unconstrained optimization problems. A well-known penalty function for this problem is given by

$$
F(x, \mu)=f(x)+\mu \frac{1}{\theta} \sum_{\ell=1}^{p}\left(h_{\ell}(x)\right)^{\theta}
$$

where $\theta>0$ is a constant and $\mu>0$ is an auxiliary penalty variable. The corresponding unconstrained optimization problem of (3) is defined as follows

$$
\text { minimize } F(x, \mu) \quad \text { s.t. } \quad x \in \mathfrak{R}^{n} \text {. }
$$


For more details about NLP problem can be found in ( [12-14], [17], [18]).

We can write the NLP problem in a system of fractional differential equations as follows:

Consider the unconstrained optimization problem (5), an approach based on fractional dynamic system can be described by the following FDEs

$$
D^{\alpha} x(t)=-\nabla_{x} F(x, \mu), \quad 0<\alpha \leq 1,
$$

with the initial conditions $x\left(t_{0}\right)=c_{i}, i=1,2, \ldots, n$.

Note that, a point $x_{e}$ is called an equilibrium point of (6) if it satisfies the right hand side of Eq.(6). Also, we can rewrite the fractional dynamic system (6) in more general form as follows

$$
D^{\alpha} x_{i}(t)=g_{i}\left(t, \mu, x_{1}, x_{2}, \ldots, x_{n}\right), \quad i=1,2, \ldots, n .
$$

The steady state solution of the non-linear system of FDEs (7) must be coincided with local optimal solution of the NLP problem (3).

\section{Derivation an approximate formula for fractional derivatives using Chebyshev series expansion}

The well known Chebyshev polynomials [25] are defined on the interval $[-1,1]$ and can be determined with the aid of the following recurrence formula

$$
\begin{aligned}
T_{n+1}(z)=2 z T_{n}(z)-T_{n-1}(z) \\
T_{0}(z)=1, \quad T_{1}(z)=z, \quad n=1,2, \ldots
\end{aligned}
$$

The analytic form of the Chebyshev polynomials $T_{n}(z)$ of degree $n$ is given by

$$
T_{n}(z)=n \sum_{i=0}^{\left[\frac{n}{2}\right]}(-1)^{i} 2^{n-2 i-1} \frac{(n-i-1) !}{(i) !(n-2 i) !} z^{n-2 i}
$$

where $[n / 2]$ denotes the integer part of $n / 2$. The orthogonality condition is

$$
\int_{-1}^{1} \frac{T_{i}(z) T_{j}(z)}{\sqrt{1-z^{2}}} d z= \begin{cases}\pi, & \text { for } i=j=0 \\ \frac{\pi}{2}, & \text { for } i=j \neq 0 \\ 0, & \text { for } i \neq j\end{cases}
$$

In order to use these polynomials on the interval $[0, L]$ we define the so called shifted Chebyshev polynomials by introducing the change of variable $z=\frac{2}{L} t-1$. The shifted Chebyshev polynomials are defined as $T_{n}^{*}(t)=T_{n}\left(\frac{2}{L} t-1\right)=T_{2 n}(\sqrt{t / L})$. The analytic form of the shifted Chebyshev polynomials $T_{n}^{*}(t)$ of degree $n$ is given by

$$
T_{n}^{*}(t)=n \sum_{k=0}^{n}(-1)^{n-k} \frac{2^{2 k}(n+k-1) !}{L^{k}(2 k) !(n-k) !} t^{k}, n=2,3, \ldots
$$

The function $x(t)$, which belongs to the space of square integrable functions on $[0, L]$, may be expressed in terms of shifted Chebyshev polynomials as

$$
x(t)=\sum_{i=0}^{\infty} c_{i} T_{i}^{*}(t)
$$

where the coefficients $c_{i}$ are given by (for $i=1,2, \ldots$ )

$$
c_{0}=\frac{1}{\pi} \int_{0}^{L} \frac{x(t) T_{0}^{*}(t)}{\sqrt{L t-t^{2}}} d t, \quad c_{i}=\frac{2}{\pi} \int_{0}^{L} \frac{x(t) T_{i}^{*}(t)}{\sqrt{L t-t^{2}}} d t .
$$

In practice, only the first $(m+1)$-terms of shifted Chebyshev polynomials are considered. Then we have

$$
x_{m}(t)=\sum_{i=0}^{m} c_{i} T_{i}^{*}(t)
$$

Theorem 3.1 (Chebyshev truncation theorem) [25] The error in approximating $x(t)$ by the sum of its first $m$ terms is bounded by the sum of the absolute values of all the neglected coefficients. If

$$
x_{m}(t)=\sum_{k=0}^{m} c_{k} T_{k}(t)
$$

then

$$
E_{T}(m) \equiv\left|x(t)-x_{m}(t)\right| \leq \sum_{k=m+1}^{\infty}\left|c_{k}\right|,
$$

for all $x(t)$, all $m$, and all $t \in[-1,1]$.

The main approximate formula of the fractional derivative of $x_{m}(t)$ is given in the following theorem.

Theorem 3.2 Let $x(t)$ be approximated by Chebyshev polynomials as (12) and also suppose $\alpha>0$, then

$$
D^{\alpha}\left(x_{m}(t)\right)=\sum_{i=\lceil\alpha\rceil}^{m} \sum_{k=\lceil\alpha\rceil}^{i} c_{i} w_{i, k}^{(\alpha)} t^{k-\alpha},
$$

where $w_{i, k}^{(\alpha)}$ is given by

$$
w_{i, k}^{(\alpha)}=(-1)^{i-k} \frac{2^{2 k} i(i+k-1) ! \Gamma(k+1)}{L^{k}(i-k) !(2 k) ! \Gamma(k+1-\alpha)} .
$$

Proof. Since the Caputo's fractional differentiation is a linear operation we have

$$
D^{\alpha}\left(x_{m}(t)\right)=\sum_{i=0}^{m} c_{i} D^{\alpha}\left(T_{i}^{*}(t)\right)
$$

Employing Eqs.(1) and (2) on the formula (9) we have

$$
D^{\alpha} T_{i}^{*}(t)=0, \quad i=0,1, \ldots,\lceil\alpha\rceil-1, \quad \alpha>0 .
$$


Also, for $i=\lceil\alpha\rceil,\lceil\alpha\rceil+1, \ldots, m$, and by using Eqs.(1) and (2), we get

$$
\begin{aligned}
D^{\alpha} T_{i}^{*}(t) & =i \sum_{k=\lceil\alpha\rceil}^{i}(-1)^{i-k} \frac{2^{2 k}(i+k-1) !}{L^{k}(i-k) !(2 k) !} D^{\alpha} t^{k} \\
& =i \sum_{k=\lceil\alpha\rceil}^{i}(-1)^{i-k} \frac{2^{2 k}(i+k-1) ! \Gamma(k+1)}{L^{k}(i-k) !(2 k) ! \Gamma(k+1-\alpha)} t^{k-\alpha}
\end{aligned}
$$

A combination of Eqs.(18), (19) and (16) leads to the desired result (15) and completes the proof of the theorem.

Theorem 3.3 The Caputo fractional derivative of order $\alpha$ for the shifted Chebyshev polynomials can be expressed in terms of the shifted Chebyshev polynomials themselves in the following form

$$
D^{\alpha}\left(T_{i}^{*}(t)\right)=\sum_{k=\lceil\alpha\rceil}^{i} \sum_{j=0}^{k-\lceil\alpha\rceil} \Theta_{i, j, k} T_{j}^{*}(t)
$$

where (for $j=0,1, \ldots$ )

$$
\Theta_{i, j, k}=\frac{(-1)^{i-k} 2 i(i+k-1) ! \Gamma\left(k-\alpha+\frac{1}{2}\right)}{h_{j} \Gamma\left(k+\frac{1}{2}\right)(i-k) ! \Gamma(k-\alpha-j+1) \Gamma(k+j-\alpha+1) L^{k}}
$$

Proof. We concern the properties of the shifted Chebyshev polynomials [25] and expanding $t^{k-\alpha}$ in Eq.(19) in the following form

$$
t^{k-\alpha}=\sum_{j=0}^{k-\lceil\alpha\rceil} c_{k j} T_{j}^{*}(t)
$$

where $c_{k j}$ can be obtained using (11) where $x(t)=t^{k-\alpha}$ then

$c_{k j}=\frac{2}{h_{j} \pi} \int_{0}^{L} \frac{t^{k-\alpha} T_{j}^{*}(t)}{\sqrt{L t-t^{2}}} d t, \quad h_{0}=2, h_{j}=1, j=1,2, \ldots$.

At $j=0$ we find

$$
c_{k 0}=\frac{1}{\pi} \int_{0}^{L} \frac{t^{k-\alpha} T_{0}^{*}(t)}{\sqrt{L t-t^{2}}} d t=\frac{1}{\sqrt{\pi}} \frac{\Gamma(k-\alpha+1 / 2)}{\Gamma(k-\alpha+1)},
$$

also, at any $j$ and using the formula (9) we can find that $c_{k j}=\frac{j}{\sqrt{\pi}} \sum_{r=0}^{j}(-1)^{j-r} \frac{(j+r-1) ! 2^{2 r+1} \Gamma(k+r-\alpha+1 / 2)}{(j-r) !(2 r) ! \Gamma(k+r-\alpha+1) L^{r}}$,

for $j=1,2, \ldots$. Employing Eqs.(19) and (21) gives $D^{\alpha}\left(T_{i}^{*}(t)\right)=\sum_{k=\lceil\alpha\rceil}^{i} \sum_{j=0}^{k-\lceil\alpha\rceil} \Theta_{i, j, k} T_{j}^{*}(t), \quad i=\lceil\alpha\rceil,\lceil\alpha\rceil+1, \ldots$, where

$\Theta_{i, j, k}=$

$\begin{cases}i \frac{(-1)^{i-k}(i+k-1) ! 2^{2 k} k ! \Gamma\left(k-\alpha+\frac{1}{2}\right)}{(i-k) !(2 k) ! \sqrt{\pi}(\Gamma(k+1-\alpha))^{2}}, & j=0 ; \\ \frac{(-1)^{i-k} i j(i+k-1) ! 2^{2 k+1} k !}{\sqrt{\pi} \Gamma(k+1-\alpha)(i-k) !(2 k) !} \times \sum_{r=0}^{j} \frac{(-1)^{j-r}(j+r-1) ! 2^{2 r} \Gamma\left(k+r-\alpha+\frac{1}{2}\right)}{(j-r) !(2 r) ! \Gamma(k+r-\alpha+1) L^{r}}, & j=1,2, \ldots\end{cases}$
After some lengthly manipulation $\Theta_{i, j, k}$ can put in the following form (for $j=0,1, \ldots$ )

$\Theta_{i, j, k}=\frac{(-1)^{i-k} 2 i(i+k-1) ! \Gamma\left(k-\alpha+\frac{1}{2}\right)}{h_{j} \Gamma\left(k+\frac{1}{2}\right)(i-k) ! \Gamma(k-\alpha-j+1) \Gamma(k+j-\alpha+1) L^{k}}$,

and this completes the proof of the theorem.

Theorem 3.4 The error $\left|E_{T}(m)\right|=\left|D^{\alpha} x(t)-D^{\alpha} x_{m}(t)\right|$ in approximating $D^{\alpha} x(t)$ by $D^{\alpha} x_{m}(t)$ is bounded by

$$
\left|E_{T}(m)\right| \leq\left|\sum_{i=m+1}^{\infty} c_{i}\left(\sum_{k=\lceil\alpha\rceil}^{i} \sum_{j=0}^{k-\lceil\alpha\rceil} \Theta_{i, j, k}\right)\right|
$$

Proof. A combination of Eqs.(10), (12) and (20) leads to

$$
\begin{aligned}
\left|E_{T}(m)\right| & =\left|D^{\alpha} x(t)-D^{\alpha} x_{m}(t)\right| \\
& =\left|\sum_{i=m+1}^{\infty} c_{i}\left(\sum_{k=\lceil\alpha\rceil}^{i} \sum_{j=0}^{k-\lceil\alpha\rceil} \Theta_{i, j, k} T_{j}^{*}(t)\right)\right|,
\end{aligned}
$$

but $\left|T_{j}^{*}(t)\right| \leq 1$, so, we can obtain

$$
\left|E_{T}(m)\right| \leq\left|\sum_{i=m+1}^{\infty} c_{i}\left(\sum_{k=\lceil\alpha\rceil}^{i} \sum_{j=0}^{k-\lceil\alpha\rceil} \Theta_{i, j, k}\right)\right|
$$

and subtracting the truncated series from the infinite series, bounding each term in the difference, and summing the bounds completes the proof of the theorem.

\section{Numerical implementation}

In order to illustrate the effectiveness of the proposed method, we implement them to solve the following system of FDEs which is generated from the non-linear programming problem.

\subsection{Optimization problem 1:}

Consider the following non-linear programming problem [26]

$$
\begin{aligned}
& \operatorname{minimize} f(x)=100\left(u^{2}-v\right)^{2}+(u-1)^{2}, \\
& \text { subject to } h(x)=u(u-4)-2 v+12=0 .
\end{aligned}
$$

The optimal solution is $x^{*}=(2,4)$, where $x=(u, v)$. For solving the above problem, we convert it to an unconstrained optimization problem with quadratic penalty function (4) for $\theta=2$, then we have $F(x, \mu)=100\left(u^{2}-v\right)^{2}+(u-1)^{2}+\frac{1}{2} \mu(u(u-4)-2 v+12)^{2}$, where $\mu \in \mathfrak{R}^{+}$is an auxiliary penalty variable. The 
corresponding non-linear system of FDEs from (6) is defined as

$D^{\alpha} u(t)=-400\left(u^{2}-v\right) u-2(u-1)-\mu(2 u-4)\left(u^{2}-4 u-2 v+12\right)$, $D^{\alpha} v(t)=200\left(u^{2}-v\right)+2 \mu\left(u^{2}-4 u-2 v+12\right), \quad 0<\alpha \leq 1$,

with the following initial conditions $u(0)=0$ and $v(0)=0$.

\section{I: Implementation of Chebyshev approximation}

Consider the system of fractional differential equations (25). In order to use the Chebyshev collocation method, we first approximate $u(t)$ and $v(t)$ as

$$
u_{m}(t)=\sum_{i=0}^{m} a_{i} T_{i}^{*}(t), \quad v_{m}(t)=\sum_{i=0}^{m} b_{i} T_{i}^{*}(t) .
$$

From Eqs.(26) and Theorem 3.2 we have

$$
\begin{array}{r}
\sum_{i=\lceil\alpha\rceil}^{m} \sum_{k=\lceil\alpha\rceil}^{i} a_{i} w_{i, k}^{(\alpha)} t^{k-\alpha}=-400\left(\left(\sum_{i=0}^{m} a_{i} T_{i}^{*}(t)\right)^{2}-\sum_{i=0}^{m} b_{i} T_{i}^{*}(t)\right) \\
\left(\sum_{i=0}^{m} a_{i} T_{i}^{*}(t)\right)-2\left(\sum_{i=0}^{m} a_{i} T_{i}^{*}(t)-1\right)-\mu\left(2 \sum_{i=0}^{m} a_{i} T_{i}^{*}(t)-4\right) \\
\left(\left(\sum_{i=0}^{m} a_{i} T_{i}^{*}(t)\right)^{2}-4\left(\sum_{i=0}^{m} a_{i} T_{i}^{*}(t)\right)-2\left(\sum_{i=0}^{m} b_{i} T_{i}^{*}(t)\right)+12\right), \\
\sum_{i=\lceil\alpha\rceil k=\lceil\alpha\rceil}^{m} \sum_{k=1}^{i} b_{i} w_{i, k}^{(\alpha)} t^{k-\alpha}=200\left(\left(\sum_{i=0}^{m} a_{i} T_{i}^{*}(t)\right)^{2}-\sum_{i=0}^{m} b_{i} T_{i}^{*}(t)\right) \\
+2 \mu\left(\left(\sum_{i=0}^{m} a_{i} T_{i}^{*}(t)\right)^{2}-4\left(\sum_{i=0}^{m} a_{i} T_{i}^{*}(t)\right)-2\left(\sum_{i=0}^{m} b_{i} T_{i}^{*}(t)\right)+12\right) .
\end{array}
$$

We now collocate Eqs.(27) and (28) at $(m+1-\lceil\alpha\rceil)$ points $t_{p}(p=0,1, \ldots, m+1-\lceil\alpha\rceil)$ as

$$
\begin{aligned}
& \sum_{i=\lceil\alpha\rceil k=\lceil\alpha\rceil}^{m} \sum_{i}^{i} a_{i} w_{i, k}^{(\alpha)} t_{p}^{k-\alpha}=-400\left(\left(\sum_{i=0}^{m} a_{i} T_{i}^{*}\left(t_{p}\right)\right)^{2}-\sum_{i=0}^{m} b_{i} T_{i}^{*}\left(t_{p}\right)\right) \\
& \left(\sum_{i=0}^{m} a_{i} T_{i}^{*}\left(t_{p}\right)\right)-2\left(\sum_{i=0}^{m} a_{i} T_{i}^{*}\left(t_{p}\right)-1\right)-\mu\left(2 \sum_{i=0}^{m} a_{i} T_{i}^{*}\left(t_{p}\right)\right. \\
& -4)\left(\left(\sum_{i=0}^{m} a_{i} T_{i}^{*}\left(t_{p}\right)\right)^{2}-4\left(\sum_{i=0}^{m} a_{i} T_{i}^{*}\left(t_{p}\right)\right)-2\left(\sum_{i=0}^{m} b_{i} T_{i}^{*}\left(t_{p}\right)\right)+12\right),
\end{aligned}
$$

$$
\begin{aligned}
& \sum_{i=\lceil\alpha\rceil k=\lceil\alpha\rceil}^{m} \sum_{k}^{i} b_{i} w_{i, k}^{(\alpha)} t_{p}^{k-\alpha}=200\left(\left(\sum_{i=0}^{m} a_{i} T_{i}^{*}\left(t_{p}\right)\right)^{2}-\right. \\
& \left.\quad \sum_{i=0}^{m} b_{i} T_{i}^{*}\left(t_{p}\right)\right)+2 \mu\left(\left(\sum_{i=0}^{m} a_{i} T_{i}^{*}\left(t_{p}\right)\right)^{2}-4\left(\sum_{i=0}^{m} a_{i} T_{i}^{*}\left(t_{p}\right)\right)\right. \\
& \left.\quad-2\left(\sum_{i=0}^{m} b_{i} T_{i}^{*}\left(t_{p}\right)\right)+12\right) .
\end{aligned}
$$

For suitable collocation points we use the roots of shifted Chebyshev polynomial $T_{m+1-\lceil\alpha\rceil}^{*}(t)$.
Also, by substituting Eq.(26) in the initial conditions $u(0)=v(0)=0$, we can find

$$
\sum_{i=0}^{m}(-1)^{i} a_{i}=0, \quad \sum_{i=0}^{m}(-1)^{i} b_{i}=0 .
$$

Equations (29) and (30), together the equations of the initial conditions (31), give $(2 m+2)$ of non-linear algebraic equations which can be solved using the Newton iteration method, for the unknowns $a_{i}$ and $b_{i}, i=0,1, \ldots, m$.

\section{II: Implementation of fractional FDM}

In this section, the fractional finite difference method with the discrete formula ( [27], [28]) is used to estimate the time $\alpha$-order fractional derivative to solve numerically the system of FDEs (25). Using ( [27], [28]) the restriction of the exact solution to the grid points centered at $x_{n}=$ $n k, n=1,2, \ldots, N$, in Eqs.(25)

$$
\begin{gathered}
\sigma_{\alpha, k} \sum_{j=1}^{n} \omega_{j}^{(\alpha)}\left(u_{n-j+1}-u_{n-j}\right)+O(k)=-400\left(u_{n}^{2}-v_{n}\right) u_{n} \\
-2\left(u_{n}-1\right)-\mu\left(2 u_{n}-4\right) \cdot\left(u_{n}^{2}-4 u_{n}-2 v_{n}+12\right) \\
\sigma_{\alpha, k} \sum_{j=1}^{n} \omega_{j}^{(\alpha)}\left(v_{n-j+1}-v_{n-j}\right)+O(k) \\
\quad=200\left(u_{n}^{2}-v_{n}\right)+2 \mu\left(u_{n}^{2}-4 u_{n}-2 v_{n}+12\right) \\
\sigma_{\alpha, k} \sum_{j=1}^{n} \omega_{j}^{(\alpha)}\left(u_{n-j+1}-u_{n-j}\right)=-400\left(u_{n}^{2}-v_{n}\right) u_{n} \\
-2\left(u_{n}-1\right)-\mu\left(2 u_{n}-4\right) \cdot\left(u_{n}^{2}-4 u_{n}-2 v_{n}+12\right)+T E_{1}(t), \\
\sigma_{\alpha, k} \sum_{j=1}^{n} \omega_{j}^{(\alpha)}\left(v_{n-j+1}-v_{n-j}\right)=200\left(u_{n}^{2}-v_{n}\right) \\
\quad+2 \mu\left(u_{n}^{2}-4 u_{n}-2 v_{n}+12\right)+T E_{2}(t),
\end{gathered}
$$

where $T E_{1}(t)$ and $T E_{2}(t)$ are the truncation terms. Thus, according to Eqs.(34) and (35), the numerical scheme is consistent, first order correct in time. The resulting finite difference equations are defined by

$$
\begin{aligned}
& \sigma_{\alpha, k} \sum_{j=1}^{n} \omega_{j}^{(\alpha)}\left(u_{n-j+1}-u_{n-j}\right)=-400\left(u_{n}^{2}-v_{n}\right) u_{n} \\
& \quad-2\left(u_{n}-1\right)-\mu\left(2 u_{n}-4\right) \cdot\left(u_{n}^{2}-4 u_{n}-2 v_{n}+12\right) \\
& \sigma_{\alpha, k} \sum_{j=1}^{n} \omega_{j}^{(\alpha)}\left(v_{n-j+1}-v_{n-j}\right)=200\left(u_{n}^{2}-v_{n}\right) \\
& \quad+2 \mu\left(u_{n}^{2}-4 u_{n}-2 v_{n}+12\right), \quad n=1,2, \ldots, N
\end{aligned}
$$

This scheme presents a non-linear system of algebraic equations. In our calculation, we used the Newton iteration method to solve this system. 


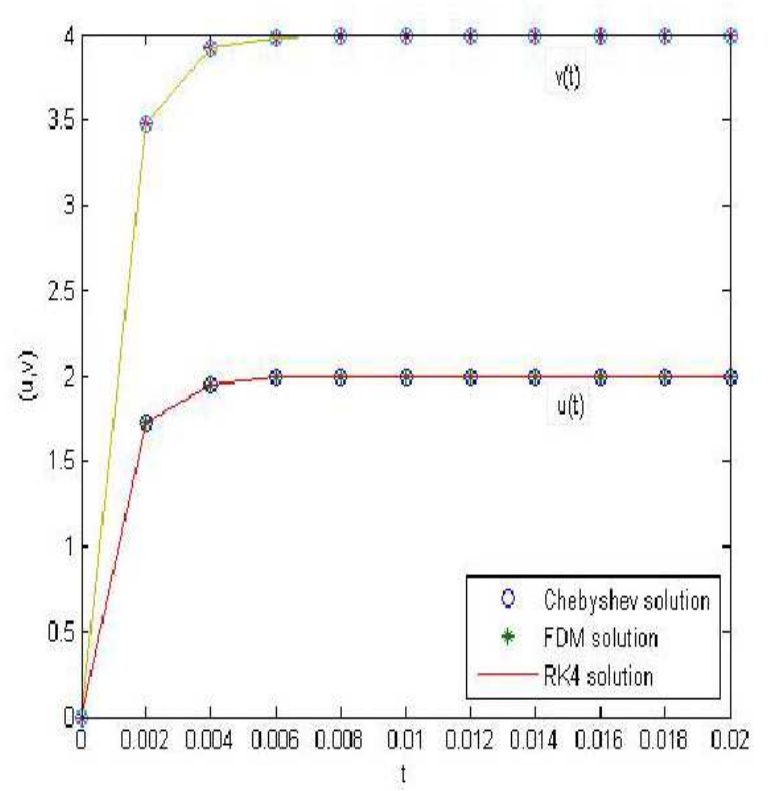

Fig. 1: The behavior of the Chebyshev collocation solution with $m=4$, FDM solution with $k=0.002$ and RK4 solution at $\alpha=1$.

In figures 1 and 2 , we presented a comparison between the approximate solution $(u(t), v(t))$ using the Chebyshev collocation method with $m=4$, numerical solution using the fractional finite difference method with $k=0.002$ and the solution using Runge-Kutta method for $\alpha=1$ and $\alpha=0.85$, respectively. From these figures, we can conclude that the obtained numerical solutions of the proposed methods are in excellent agreement with those obtained from Runge-Kutta method.

\subsection{Optimization problem 2:}

Consider the equality constrained optimization problem [26]

$$
\begin{gathered}
\text { minimize } \begin{aligned}
f(x)= & \left(x_{1}-1\right)^{2}+\left(x_{1}-x_{2}\right)^{2}+\left(x_{2}-x_{3}\right)^{2} \\
& +\left(x_{3}-x_{4}\right)^{4}+\left(x_{4}-x_{5}\right)^{4},
\end{aligned} \\
\text { subject to } h_{1}(x)=x_{1}+x_{2}^{2}+x_{3}^{3}-2-3 \sqrt{2}=0, \\
h_{2}(x)=x_{2}-x_{3}^{2}+x_{4}+2-2 \sqrt{2}=0, \\
h_{3}(x)=x_{1} x_{5}-2=0 .
\end{gathered}
$$

The solution of $(38)$ is $x^{*} \cong(1.19,1.362,1.47,1.64,1.68)$ and this is not an exact solution. For solving the above problem, we convert it to an unconstrained optimization problem with quadratic penalty function (4) for $\theta=2$, then

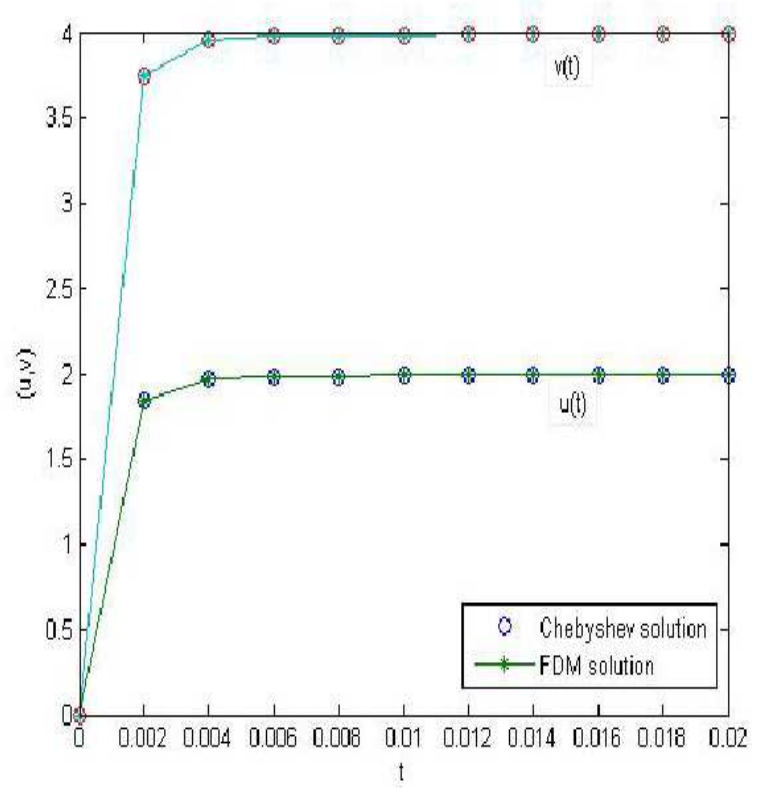

Fig. 2: The behavior of the Chebyshev collocation solution with $m=4$ and FDM solution with $k=0.002$ at $\alpha=0.85$.

Table 1: The numerical solution of the system (40) using the Chebyshev collocation method at $\alpha=1$.

\begin{tabular}{|c|c|c|c|c|c|}
\hline$t$ & $x_{1}(t)$ & $x_{2}(t)$ & $x_{3}(t)$ & $x_{4}(t)$ & $x_{5}(t)$ \\
\hline 0 & 2 & 2 & 2 & 2 & 2 \\
2 & 1.19101 & 1.35954 & 1.47404 & 1.64153 & 1.67921 \\
10 & 1.19108 & 1.36252 & 1.47278 & 1.63476 & 1.67914 \\
15 & 1.19109 & 1.36253 & 1.47277 & 1.63474 & 1.67913 \\
20 & 1.19109 & 1.36253 & 1.47277 & 1.63474 & 1.67913 \\
30 & 1.19109 & 1.36253 & 1.47277 & 1.63474 & 1.67913 \\
\hline
\end{tabular}

we have

$$
F(x, \mu)=f(x)+\frac{1}{2} \mu \sum_{\ell=1}^{3}\left(h_{\ell}(x)\right)^{2},
$$

where $\mu \in \mathfrak{R}^{+}$is an auxiliary penalty variable. The corresponding non-linear system of FDEs from (6) is defined as

$$
D^{\alpha} x(t)=-\nabla f(x)-\mu \nabla h(x) h(x), \quad 0<\alpha \leq 1,
$$

with the following initial conditions $x(0)=(2,2,2,2,2)^{T}$ that is not feasible.

The obtained numerical results of the problem (40) using the proposed methods are presented in tables 1-5, where in table 1 , we presented the numerical solution $x(t)=\left(x_{1}(t), x_{2}(t), \ldots, x_{5}(t)\right)$ using Chebyshev collocation method with $m=5$ at $\alpha=1$ and in table 2, we presented the numerical solution using the fractional FDM with 
Table 2: The numerical solution of the system (40) using the fractional FDM at $\alpha=1$.

\begin{tabular}{|c|c|c|c|c|c|}
\hline$t$ & $x_{1}(t)$ & $x_{2}(t)$ & $x_{3}(t)$ & $x_{4}(t)$ & $x_{5}(t)$ \\
\hline 0 & 2 & 2 & 2 & 2 & 2 \\
2 & 1.19101 & 1.35954 & 1.47404 & 1.64153 & 1.67920 \\
10 & 1.19108 & 1.36252 & 1.47278 & 1.63476 & 1.67914 \\
15 & 1.19109 & 1.36253 & 1.47277 & 1.63474 & 1.67913 \\
20 & 1.19109 & 1.36253 & 1.47277 & 1.63474 & 1.67913 \\
30 & 1.19109 & 1.36253 & 1.47277 & 1.63474 & 1.67913 \\
\hline
\end{tabular}

Table 3: The numerical solution of the system (40) using the RK4 method at $\alpha=1$.

\begin{tabular}{|c|c|c|c|c|c|}
\hline$t$ & $x_{1}(t)$ & $x_{2}(t)$ & $x_{3}(t)$ & $x_{4}(t)$ & $x_{5}(t)$ \\
\hline 0 & 2 & 2 & 2 & 2 & 2 \\
2 & 1.19101 & 1.35954 & 1.47404 & 1.64153 & 1.67921 \\
10 & 1.19108 & 1.36252 & 1.47278 & 1.63476 & 1.67914 \\
15 & 1.19109 & 1.36253 & 1.47277 & 1.63474 & 1.67913 \\
20 & 1.19109 & 1.36253 & 1.47277 & 1.63474 & 1.67913 \\
30 & 1.19109 & 1.36253 & 1.47277 & 1.63474 & 1.67913 \\
\hline
\end{tabular}

Table 4: The numerical solution of the system (40) using the Chebyshev collocation method at $\alpha=0.85$.

\begin{tabular}{|c|c|c|c|c|c|}
\hline$t$ & $x_{1}(t)$ & $x_{2}(t)$ & $x_{3}(t)$ & $x_{4}(t)$ & $x_{5}(t)$ \\
\hline 0 & 2 & 2 & 2 & 2 & 2 \\
2 & 1.19893 & 1.36922 & 1.46874 & 1.61608 & 1.66808 \\
10 & 1.19109 & 1.36253 & 1.47277 & 1.63474 & 1.67913 \\
15 & 1.19109 & 1.36253 & 1.47277 & 1.63474 & 1.67913 \\
20 & 1.19109 & 1.36253 & 1.47277 & 1.63474 & 1.67913 \\
30 & 1.19109 & 1.36253 & 1.47277 & 1.63474 & 1.67913 \\
\hline
\end{tabular}

Table 5: The numerical solution of the system (40) using the fractional FDM at $\alpha=0.85$.

\begin{tabular}{|c|c|c|c|c|c|}
\hline$t$ & $x_{1}(t)$ & $x_{2}(t)$ & $x_{3}(t)$ & $x_{4}(t)$ & $x_{5}(t)$ \\
\hline 0 & 2 & 2 & 2 & 2 & 2 \\
2 & 1.19893 & 1.36922 & 1.46874 & 1.61608 & 1.66808 \\
10 & 1.19109 & 1.36253 & 1.47277 & 1.63473 & 1.67913 \\
15 & 1.19109 & 1.36253 & 1.47277 & 1.63473 & 1.67913 \\
20 & 1.19109 & 1.36253 & 1.47277 & 1.63473 & 1.67913 \\
30 & 1.19109 & 1.36253 & 1.47277 & 1.63473 & 1.67913 \\
\hline
\end{tabular}

$k=0.002$ at $\alpha=1$ and in table 3, we presented the numerical solution using the fourth order Runge-Kutta method. But in tables 4 and 5, we presented the numerical solution of the same system (40) with $\alpha=0.85$ using the two proposed methods, respectively. From these tables, we can conclude that our solutions of the proposed methods are in excellent agreement with the solution using RK4 method.

\section{Conclusion and remarks}

In this article, we implemented an efficient numerical method for solving the system of FDEs which is generated from the NLP problem. The fractional derivative is considered in the Caputo sense. The properties of the Chebyshev polynomials are used to reduce the system of fractional differential equations to the solution of system of algebraic equations. It is evident that the overall errors can be made smaller by adding new terms from the series (26). The convergence analysis of the proposed method and derivation an upper bound of the error are introduced. From illustrative examples, it can be seen that the proposed numerical approach can obtain very accurate and satisfactory results. The numerical comparison among the fourth order Runge-Kutta $(\alpha=1)$ and the solution obtained using finite difference method with the proposed methods shows that our technique perform rapid convergence to the optimal solutions of the optimization problems. Also, from the obtained numerical results we can conclude that our results are in excellent agreement with the exact solution and those from the RK4 method. All numerical results are obtained using Matlab.

\section{Acknowledgement}

The authors are very grateful to the anonymous referees for carefully reading the paper and for their comments and suggestions that improved the paper.

\section{References}

[1] K. B. Oldham and J. Spanier, The Fractional Calculus, Academic Press, New York, (1974).

[2] I. Podlubny, Fractional Differential Equations, Academic Press, New York, (1999).

[3] R. L. Bagley and P. J. Torvik, J. Appl. Mech., 51, 294-298 (1984).

[4] M. M. Meerschaert and C. Tadjeran, Appl. Numer. Math., 56, 80-90 (2006).

[5] N. H. Sweilam, M. M. Khader and R. F. Al-Bar, Physics Letters A, 371, 26-33 (2007).

[6] I. Hashim, O. Abdulaziz and S. Momani, Communications in Nonlinear Science and Numerical Simulations , 14, 674-684 (2009).

[7] M. M. Khader, Communications in Nonlinear Science and Numerical Simulations, 16, 2535-2542 (2011).

[8] M. M. Khader, N. H. Sweilam and A. M. S. Mahdy, J. of Applied Mathematics and Bioinformatics, 1, 1-12 (2011).

[9] M. M. Khader and A. S. Hendy, International Journal of Pure and Applied Mathematics, 74, 287-297 (2012).

[10] M. M. Khader, Arab Journal of Mathematical Sciences, 18, 61-71 (2012).

[11] N. H. Sweilam, M. M. Khader and A. M. S. Mahdy, Journal of Applied Mathematics, 1-14 (2012).

[12] F. Evirgen and N. Özdemir, J. Computational Nonlinear Dynamics, 6, (2010).

[13] F. Evirgen and N. Özdemir, Fractional Dynamics and Control, 145-155 (2012).

[14] N. H. Sweilam, M. M. Khader and A. M. S. Mahdy, Journal of Fractional Calculus and Applications, 15, 1-12 (2012). 
[15] M. Abramowitz and I.A. Stegun, Handbook of mathematical functions, Dover, New York, (1964).

[16] N. H. Sweilam and M. M. Khader, ANZIAM, 51, 464-475 (2010).

[17] D. G. Luenberger, Introduction to Linear and Nonlinear Programming. Addison-Wesley, California, (1973).

[18] W. Sun and Y. Yuan, Optimization Theory and Methods: Nonlinear Programming, Springer-Verlag, New York, (2006).

[19] K. J. Arrow, L. Hurwicz and H. Uzawa, Studies in Linear and Non-Linear Programming, Stanford University Press, California, (1958).

[20] A. V. Fiacco and G. P. Mccormick, Nonlinear programming: sequential unconstrained minimization techniques, John Wiley, New York, (1968).

[21] H. Yamashita, Math. Program, 1, 155-168 (1976).

[22] G. D. Smith, Numerical Solution of Partial Differential Equations, Oxford University Press, (1965).

[23] N. H. Sweilam, M. M. Khader and A.M. Nagy, Journal of Computional and Applied Mathematics, 235, 2832-2841 (2011).

[24] S. Samko, A. Kilbas and O. Marichev, Fractional Integrals and Derivatives: Theory and Applications, Gordon and Breach, London, (1993).

[25] M. A. Snyder, Chebyshev Methods in Numerical Approximation, Prentice-Hall, Inc. Englewood Cliffs, N. J. (1966) .

[26] K. Schittkowski, More Test Examples For Nonlinear Programming Codes, Springer-Verlag, Berlin, (1987).

[27] A. Diego Murio, Computers and Mathematics with Applications, 56, 1138-1145 (2008).

[28] N. H. Sweilam, M. M. Khader and A. M. S. Mahdy, Journal of Fractional Calculus and Applications, 2, 1-9 (2012).

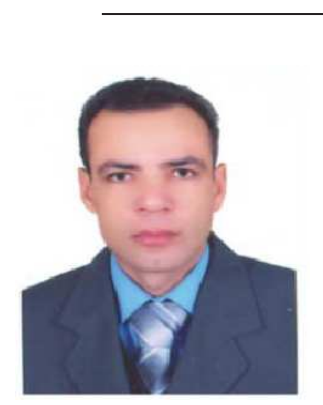

\begin{abstract}
Mohamed M. Khader received the $\mathrm{PhD}$ degree in Mathematics DepartmentFaculty of Science-Benha University-Egypt (2009). His research interests are in the areas of Pure Mathematics including the mathematical methods and numerical techniques for solving FDEs.
\end{abstract}

He has published research articles in reputed international journals of mathematical. He is referee of mathematical journals.

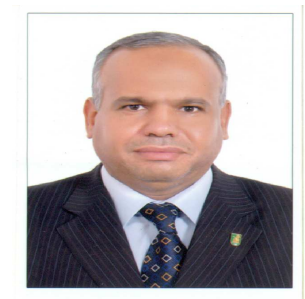

\section{Nasser H. Sweilam}

is professor of numerical analysis at the Department of Mathematics, Faculty of Science, Cairo University. He was a channel system Ph.D. student between Cairo University, Egypt, and TU-Munich, Germany. $\mathrm{He}$ received his Ph.D. in "Optimal Control of Variational Inequalities, the
Dam Problem". He is the Head of the Department of Mathematics, Faculty of Science, Cairo University, science May 2012. He is referee and editor of several international journals, in the frame of pure and applied Mathematics. His main research interests are numerical analysis, optimal control of differential equations, fractional and variable order calculus, bio-informatics and cluster computing, ill-posed problems.

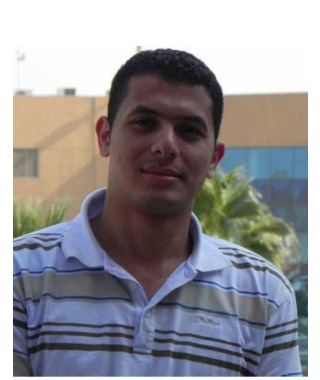

\section{Amr M. S. Mahdy}

received the $\mathrm{PhD}$ degree in Mathematics Department-Faculty of Science, Zagazig University (2013). His research interests are in the areas of Pure Mathematics including the mathematical methods and numerical techniques for solving fractional differential equations. He has published research articles in reputed international journals of mathematical. 